\title{
Leiomyosarcoma of the ovary in a young female guinea pig - a case report
}

\author{
Radka Lukášovál, ${ }^{1,2}$ Eva Bártová ${ }^{1}$, Miša Škorič ${ }^{3}$ \\ ${ }^{1}$ University of Veterinary and Pharmaceutical Sciences, Faculty of Veterinary Hygiene and Ecology, \\ Department of Biology and Wildlife Diseases, Brno, Czech Republic \\ ${ }^{2}$ Veterinary Clinic Anvet, Brno, Czech Republic, \\ ${ }^{3}$ University of Veterinary and Pharmaceutical Sciences, Faculty of Veterinary Medicine, \\ Department of Pathological Morphology and Parasitology, Brno, Czech Republic
}

Received February 9, 2017

Accepted October 2, 2017

\begin{abstract}
This case report presents a one-year old pet female smooth-coated guinea pig with a diagnosis of leiomyosarcoma of the left ovary with locally aggressive behaviour. The animal had been showing lethargy and alopetic dermatosis for a few weeks. The mass in the area of the left ovary was suspected based on palpation, $x$-ray, ultrasonography of the abdominal cavity and the presence of the mass in the abdomen was confirmed by probatory laparotomy. Since the lesion was inoperable due to its size and infiltrative growth, the animal was euthanized. Leiomyosarcoma of the ovary with infiltrative growth into spleen and pancreas was diagnosed based on histopathological examination and the diagnosis was proved by immunohistochemical staining for the presence of smooth muscle actin. This is the first described case of this type of tumour in young intact female guinea pig. The case report could contribute to better understanding and knowledge of the occurrence of neoplastic changes of the ovary in guinea pigs especially for better treatment management and prevention of reproductive tract diseases.
\end{abstract}

Ovarian tumour, ovarian cyst, reproductive problem, leiomyosarcoma

Sommerey et al. (2004) proved that spontaneous tumours in general are relatively rare in guinea pigs ( $15 \%$ of 689 animals tested) with higher prevalence in older animals $(39 \%$ of animals were $>$ four years old). Leiomyosarcoma is a malignant neoplasm with uncertain pathogenesis. Rivas et al. (2014) suggested an origin of ovarian leiomyosarcoma from the smooth muscle of the blood vessels of the cortical stroma, as well as from the muscles around the corpus luteum and ovarian follicles, muscle fibres attached to the ovarian ligament, the remainder of Wolff's duct, totipotent stem cells of the ovary, teratoma cells that have differentiated, or in smooth muscle cells that migrate from the uterus. The primary leiomyosarcoma was noticed e.g. in the heart of a three-year-old female guinea pig (Vogler et al. 2012), however, to our knowledge there is no report of ovarian leiomyosarcoma in this animal species. In women, the most common manifestation of leiomyomas is uterine and these neoplasms are called fibroids; ovarian leiomyosarcomas belong to highly aggressive tumours with hormonal influence (Fletcher et al. 2002; Divya and Srinivasamurthy 2014). In guinea pigs, cystic ovarian diseases, such as cystic rete ovarii, are also affected by hormones and are more often observed in aged females (Paterson 2006). Serous cysts are normal components of cyclic ovary in guinea pig (Shi et al. 2002). The aetiology of ovarian cysts is unknown, but a certain role may be played by oestrogenic substances in hay. Cysts are usually asymptomatic, however, as they increase in size (can be up to $10 \mathrm{~cm}$ in size and fill in a large part of the abdominal cavity), non-pruritic alopecia, abdominal enlargement and infertility may be observed. Cystic ovaries are often associated with concurrent diseases in the reproductive tract (Keller et al. 1987; Paters on 2006). Diagnosis of ovarian cysts is based on history, abdominal palpation, radiography, and ultrasonography. 
The aim of this study was to report a rare case of ovarian leiomyosarcoma in the guinea pig.

\section{Case presentation}

A 13-month-old female smooth-coated guinea pig was presented to the Veterinary Clinic for Small Animals for alopecic skin lesions (Plate IV, Fig. 1). The owners first observed weakness and lethargy, and later also a skin problem (symmetrical alopecic non-pruritic lesions on the back) lasting for a few weeks. The animal was afebrile with the body mass index 2 , dehydrated with mucous membranes of normal colour, and palpable peripheral lymph nodes of normal size and structure. The skin was not inflamed, but with large non-pruritic alopecic lesions on the back extending up to the ventral part of the abdomen. A firm structure palpated on the left side of the abdomen at a size of approximately $5 \mathrm{~cm}$ in diameter was suspected to be a neoplasm. Radiography and ultrasound confirmed the suspicion of neoplasm of the left ovary. The owners refused to use other non-invasive diagnostic methods; they accepted only probatory laparotomy and surgical extirpation of the neoplasm. The animal was anaesthetized i.m. with a combination of medetomidine (Cepetor, CP-Pharma, Burgdorf, Germany, $0.3 \mathrm{mg} / \mathrm{kg}$ ) and ketamine (Narkamon, Bioveta, Ivanovice na Hané, Czech Republic, $8 \mathrm{mg} / \mathrm{kg}$ ). Prokinetics, vitamins, antibiotics and fluid therapy were used to stabilize the animal during and after anaesthesia. The abdomen was opened in the region of linea alba with the finding of ascites and a large neoplasm $(6 \times 4 \times 4 \mathrm{~cm}$ in size $)$ on the left ovary intervening from the left uterus cranially under the left hepatic lobe (Plate IV, Fig. 2). The infiltrative growth of the neoplasia into the muscles of the abdominal wall and caudal ribs and adhesions with the mesenterium and peritoneum were evident (Plate V, Fig. 3). Another finding was a nodular neoplasia at a size of $1 \mathrm{~cm}$ in diameter in the splenic ligamentum (Plate V, Fig. 4) and small cysts on the right ovary. Both the uterine bodies and the cervix uteri were hyperplastic. Since this finding was inoperable, the animal was euthanized with intracardial application of $1 \mathrm{ml}$ T61 (Intervet International B.V., Boxmeer, Neederland, embutramidum, mebezonii iodidum, tetracaini hydrochloridum).

Subsequently, necropsy was performed, collecting the neoplastic tissue specimens for a histopathological examination (Plate VI, Fig. 5). The tissue samples were fixed in buffered $10 \%$ neutral formalin, dehydrated, embedded in paraffin wax, sectioned on a microtome at a thickness of $4 \mu \mathrm{m}$ and stained with haematoxylin and eosin, and also stained with smooth muscle actin antibody. Examination of the mass in the region of the ovary revealed neoplastic proliferation of spindle-shaped smooth muscle cells in the sparse connective tissues. The tumour was highly cellular, with cells containing elongated nuclei. Neoplastic cells formed interweaving bands and streams. There were multifocally observed cellular abnormalities, anisocytosis, anisokaryosis, prominent nucleoli, and mitotic figures. The mitotic index in the neoplastic mass was 5 mitoses per 10 high power fields (Plate VII, Fig. 6A). On the periphery of the tumour, there was presence of focal lymphoplasmacytic and mixed inflammatory reaction, and infiltrative growth of tumour cells into the parenchyma of the adjacent organs - pancreas (Plate VII, Fig. 6B), spleen, and splenic ligament with a formation of irregularly nodular mass within the connective tissue of ligament. Immunohistochemistry showed positivity of neoplastic cells for smooth muscle actin (Plate VIII, Fig. 7A and 7B). Based on the histopathological findings and immunohistochemistry, the tumour was diagnosed as an ovarian leiomyosarcoma with infiltrative growth into the parenchyma of the pancreas, spleen, and splenic ligament. A proof of oestrogen and progesteron receptors could help specify the diagnosis of this type of tumour. 


\section{Discussion}

The most common lesions in the ovaries of guinea pigs are ovarian cysts. They were described e.g. in $30 \%$ of 689 guinea pigs (Sommerey et al. 2004), and the high prevalence $(76 \%)$ was recorded in the age category from 2 to 4 years (Paterson 2006; Preetha et al. 2011). In our case, neoplasia of the left ovary with local invasiveness into the pancreas, lien and ligament, and small cysts on the right ovary were diagnosed in a 13-month-old guinea pig. In the initial stages of the cystic ovarian disease, the affected animals are usually asymptomatic, but it is followed with progressive development of abdominal distension and bilateral symmetrical non-pruritic alopecia (Paterson 2006). Because of the study by Preetha et al. (2011), the relationship between the incipient endocrine disorder and morphological changes in the other parts of the reproductive tract was proved, often leading to infertility. There is a possibility also in our case that tumour changes were primarily caused by the hormonal activity of the ovarian cysts from the right ovary, although we do not have any information about potential infertility. The owner did not observe any problems with sexual behaviour or abdominal pain. It is unknown whether the animal was without these clinical signs or they were just unnoticed by owners.

Clinical examination, palpation of abdomen, $\mathrm{x}$-ray and ultrasonography led to the suspected diagnosis of neoplasia in this case. Other non-invasive techniques (cytology, magnetic resonance and computer tomography) to specify the diagnosis were refused by owners, who elected probatory laparotomy to confirm the suspected diagnosis.

Leiomyomas are the most common tumour of the reproductive tract in aged female guinea pigs followed by uterine adenomas and rarely, uterine leiomysarcomas. Most of them are frequently observed in conjunction with cystic rete ovarii (Veiga-Pargua et al. 2016). Other tumours of the reproductive tract (ovarian teratoma and a cavernous haemangioma) were also described in guinea pigs at the mean age of 47.6 months (Field et al. 1989). Although these tumours are considered to be extremely rare in young animals, a case of ovarian teratoma was described in a one-year-old virgin female (Frisk et al. 1978), similarly as in our case.

It is assumed that in guinea pigs, there is a relationship between the hormonal activity and the formation of this type of tumour in the uterus, and more specifically the ovaries, similarly as in human medicine. Similar theory was mentioned in the study by Veiga Parga et al. (2016) who found hormone receptors as a possible component of uterine smooth muscle tumours in guinea pigs. It suggests that the hormones may play an important role in the development of these tumours. However, we did not perform examination for proving these receptors in this case. To sum up, the possible life-threatening ovarian changes could be prevented by ovariectomy or ovariohysterectomy at a young age, immediately after reaching sexual maturity, even in cases in which ovaries are still without pathological changes.

\section{References}

Sommerey CC, Kohler K, Reinacher M 2004: Diseases of guinea pigs from the pathologist's point of view. Tierarztl Prax Ausg K Kleintiere Heimtiere 32: 377-383

Rivas G, Bonilla C, Rubiano J, Arango N 2014: Primary leiomyosarcoma of the ovary: A case report. CRCM 3: 192-196

Vogler BR, Vetsch E, Wernick MB, Sydler T, Wiederkehr DD 2012: Primary leiomyosarcoma in the heart of a guinea pig. J Comp Pathol 147: 452-45

Fletcher CDM, Unni KK, Mertens F 2002: Classification of tumours. Pathology and genetics of soft tissue and bone. World Health Organisation. IARC Press: Lyon, France, pp 131-133

Divya NS, Srinivasamurthy V 2014: Myxoid leiomyosarcoma of ovary - A rare case report. J Clin Diagn Res 8: FD05-FD06

Paterson S 2006: Skin diseases of exotic pets. Blackwell Science Ltd., pp 242-244

Shi FX, Petroff BK, Herath CB, Ozawa M, Watanabe G, Taya K 2002: Serous cysts are a benign component of the cyclic ovary in the guinea pig with an incidence dependent upon inhibin bioactivity. J Vet Med Sci 64: 129-135 
Keller LSF, Griffith JW, Lang CM 1987: Reproductive failure associated with cystic rete ovarii in guinea pigs. Vet Pathol 24: 335-339

Preetha SP, Thangapandiyan M, Selvarai J, Devi RG, Roy P, Baegan S, Manohar BM 2011: Cystic ovarian disease in a laboratory guinea pig - a case report. Indian J Anim Res 45: 228-229

Veiga-Parga T, La Perle KM, Newman SJ 2016: Spontaneous reproductive pathology in female guinea pigs. J Vet Diagn Invest 28: 656-661

Field KJ, Griffith JW, Lang CM 1989: Spontaneous reproductive tract leiomyomas in aged guinea-pigs. J Comp Pathol 101: 287-294

Frisk CS, Wagner JE, Doyle RE 1978: An ovarian teratoma in a guinea pig. Lab Anim Sci 28: 199-201 
Plate IV

Lukášová R. et al.: Leiomyosarcoma of the ovary... pp. 269-272

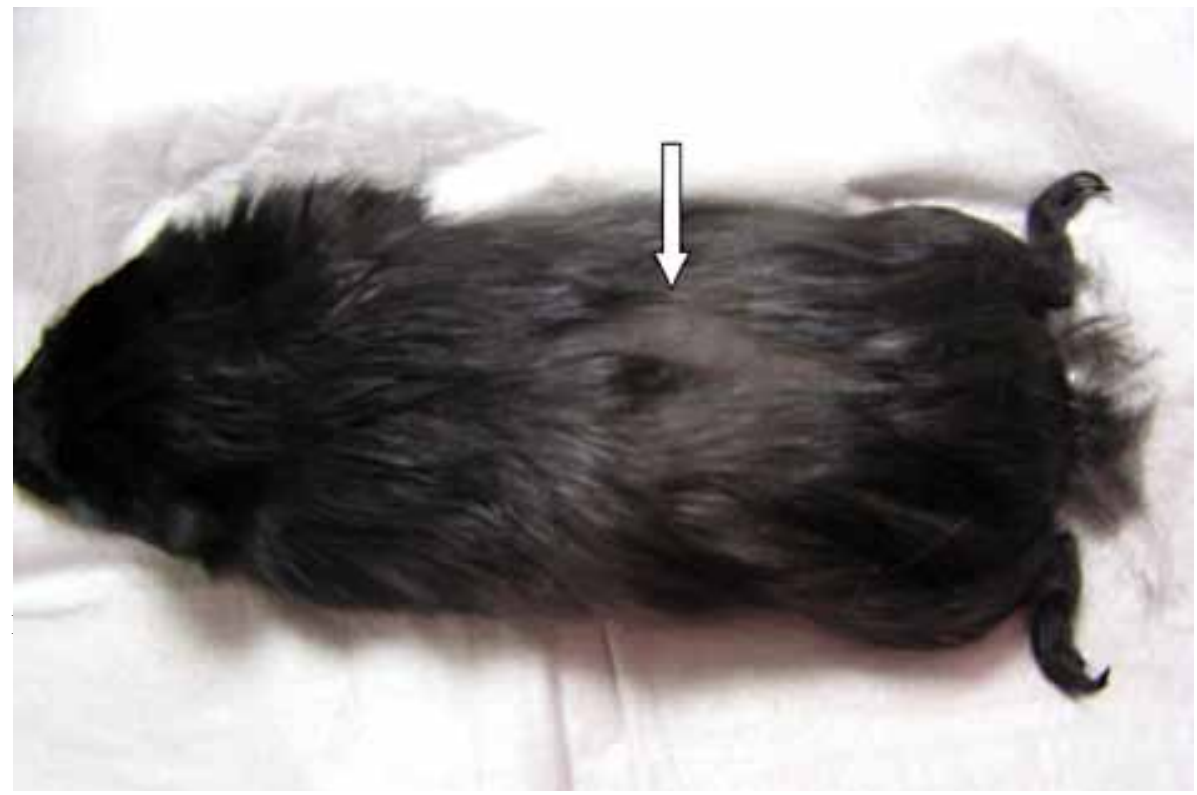

Fig. 1. Guinea pig with large alopetic lesions (white arrow) on the back extending up to the ventral part of the abdomen.

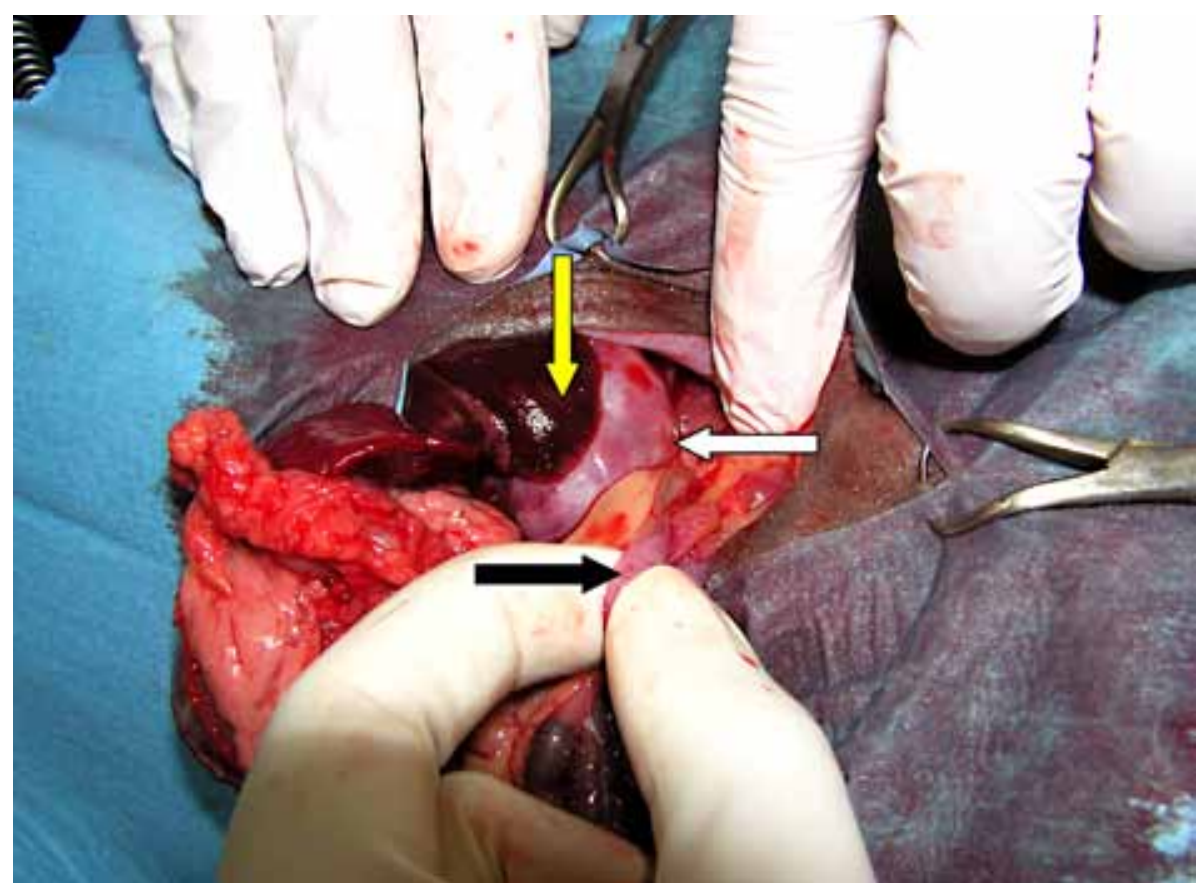

Fig. 2. Probatory laparotomy in the guinea pig with a neoplasm of the left ovary (white arrow) of the size 6 $\times 4 \mathrm{~cm}$ and intervening from the left uterus (black arrow) cranially under the left hepatic lobe (yellow arrow) 


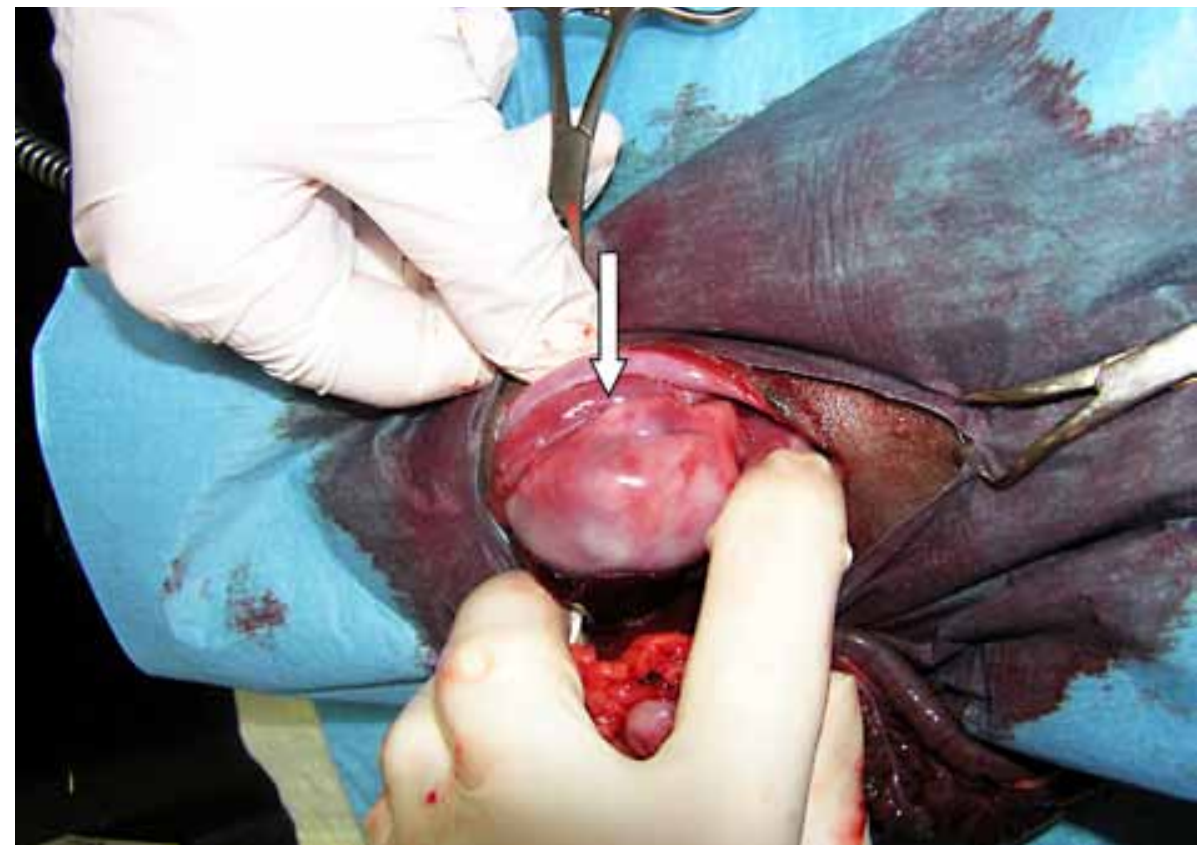

Fig. 3. Probatory laparotomy in the guinea pig with a neoplasm of the left ovary, infiltrative growth of neoplasia to the muscles of the abdominal wall and caudal ribs and adhesions with peritoneum (white arrow)

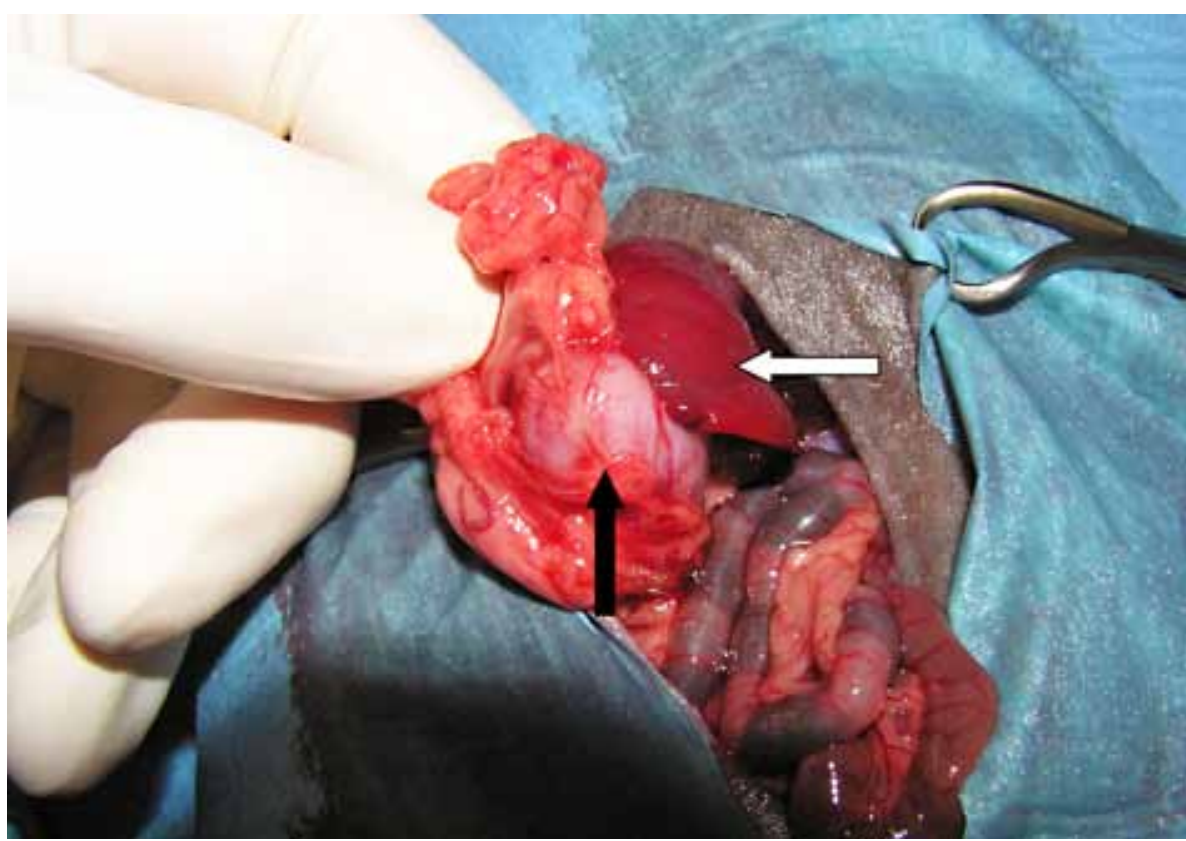

Fig. 4. Probatory laparotomy in the guinea pig with a nodular neoplasia of the size $1 \mathrm{~cm}$ in the splenic ligamentum (black arrow), and spleen (white arrow). 


\section{Plate VI}

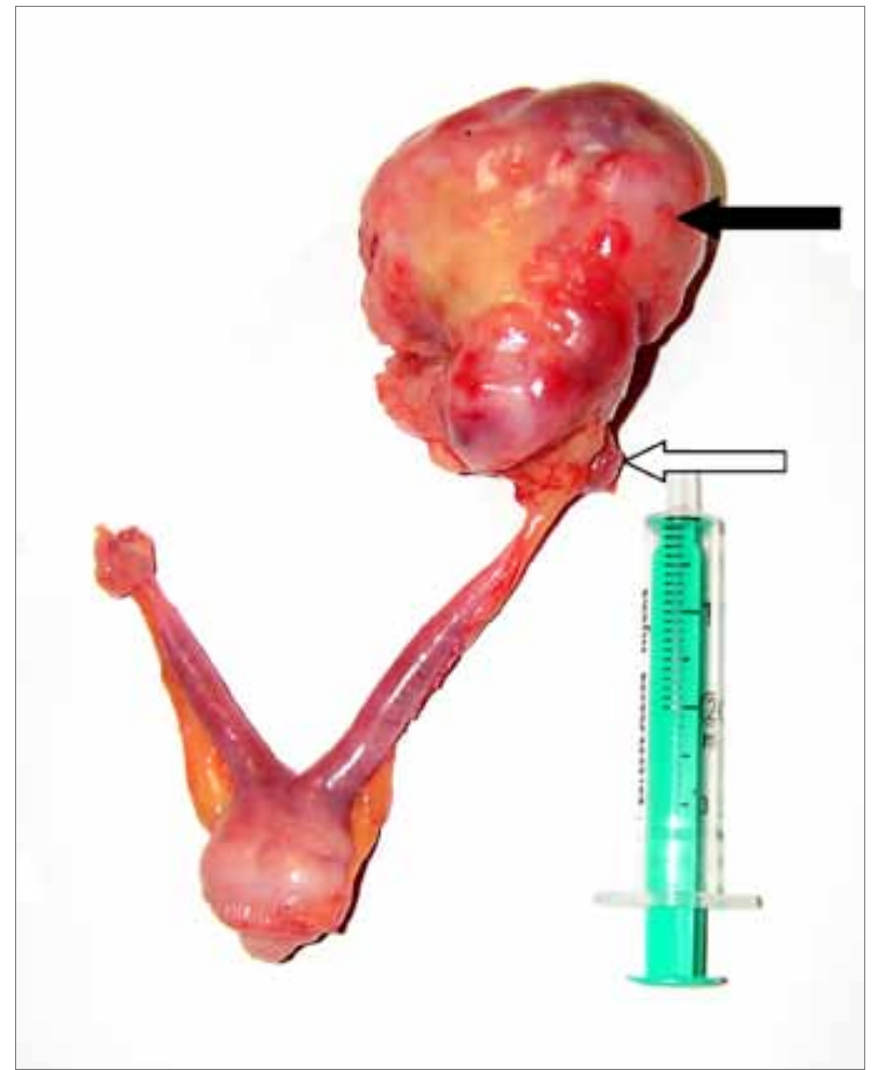

Fig. 5. The reproductive tract of the female guinea pig collected for histopathological examination with the left ovary tissue (white arrow) and the neoplasm of the left ovary (black tissue). 

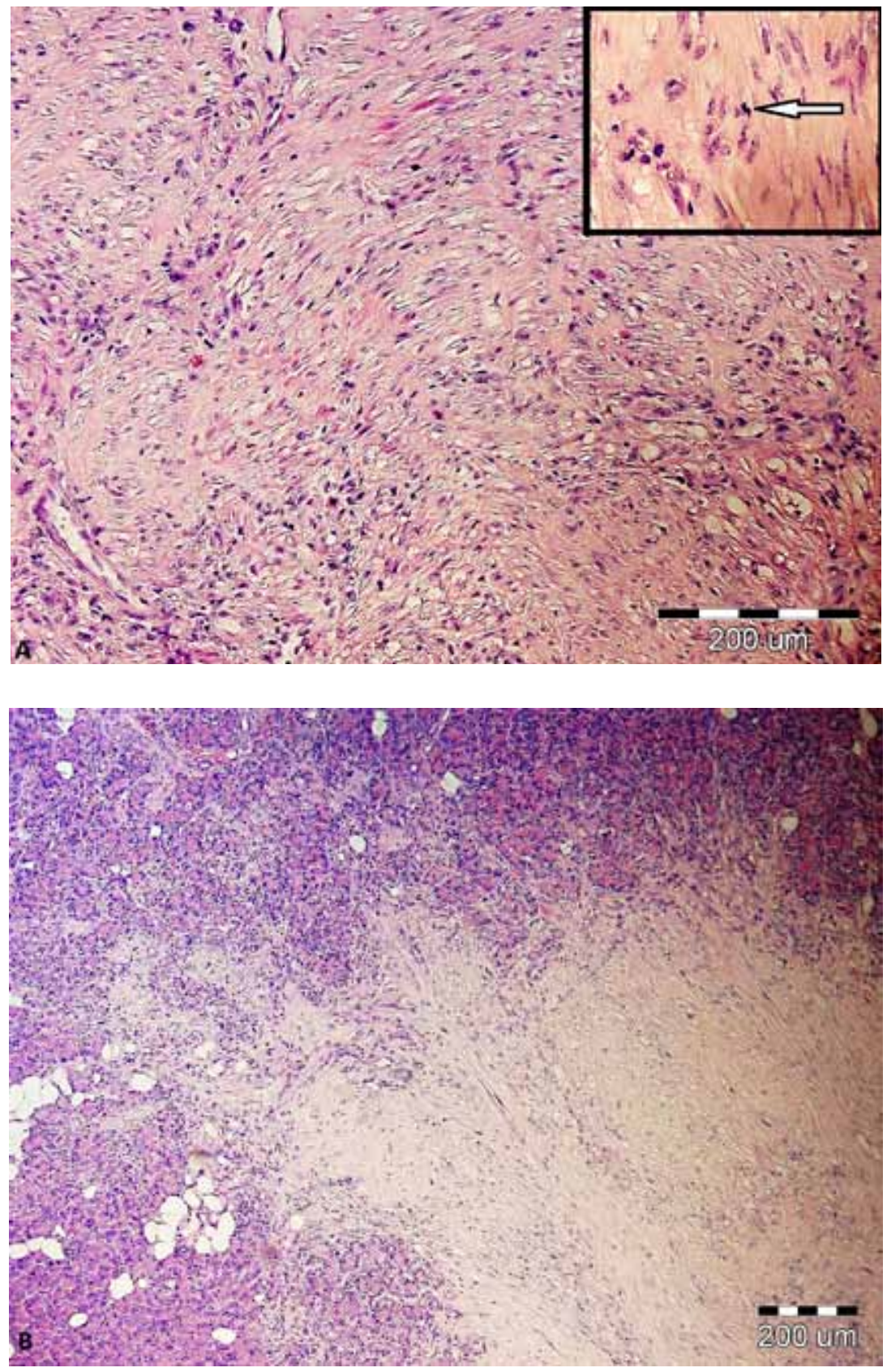

Fig. 6. Ovarian leiomyosarcoma in the guinea pig: A - spindle-shaped neoplastic cells with marked abnormalities, hypercellularity, anisocytosis, anisokaryosis, and mitotic figures (H\&E, $\times 200)$, figure with mitosis details (white arrow) in the right corner (H\&E, $\times 600)$, B - infiltrative growth of neoplastic cells on the periphery into the pancreatic parenchyma and adjacent abdominal fat tissue $(\mathrm{H} \& \mathrm{E}, \times 100)$. 

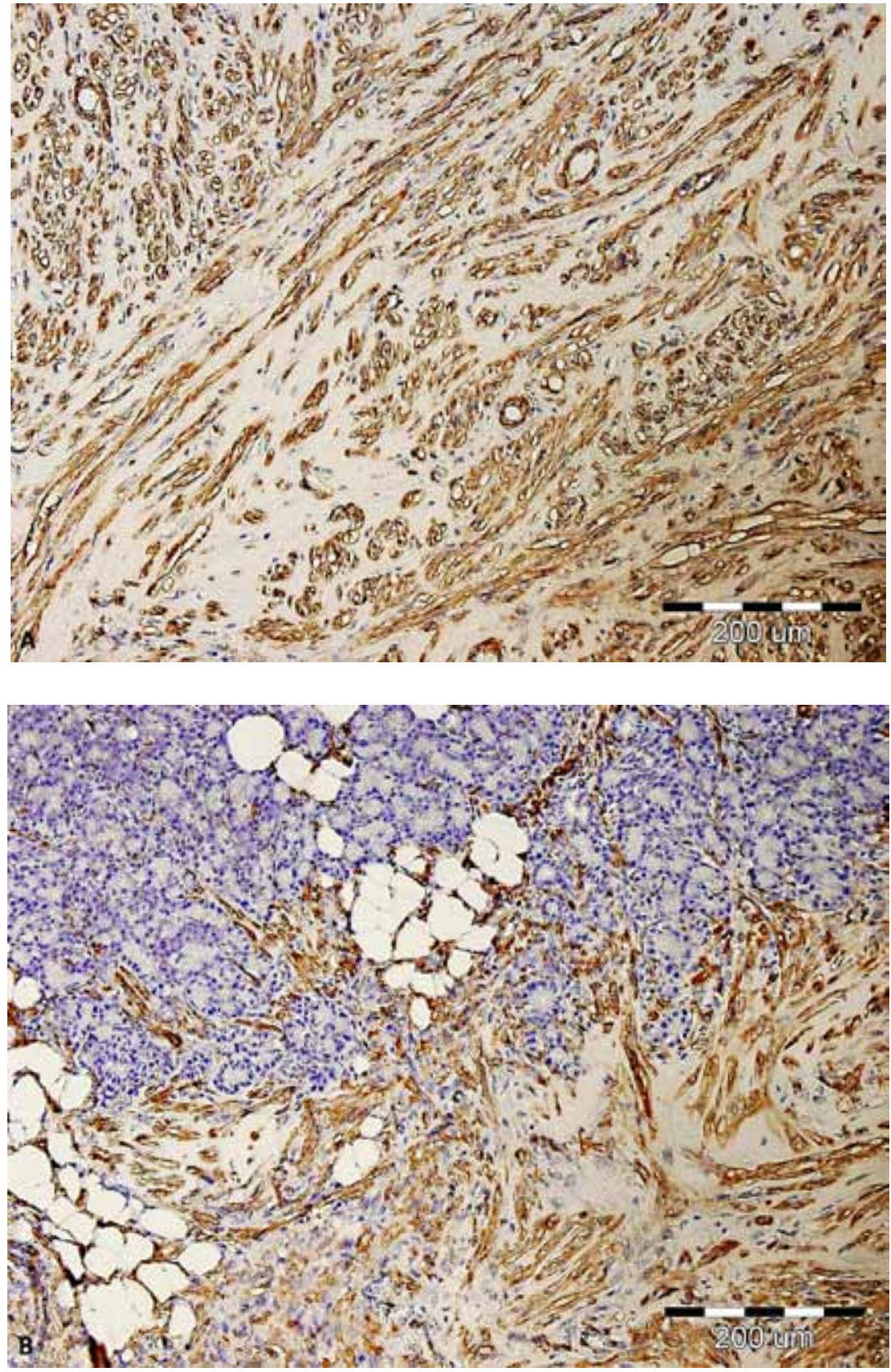

Fig. 7. Immunohistochemical staining of the tumour: A - ovarian leiomyosarcoma, smooth muscle actin positivity (SMA, $\times 200)$; B - invasion of the tumour into the pancreas. Smooth muscle actin positivity (SMA, × 200). 